\title{
NOUVELLE
}

\section{Changements globaux et infections parasitaires à mollusques vecteurs}

\section{Un schistosome hybride atteint l'Europe}

Jérôme Boissier ${ }^{1}$, Julien Kincaid-Smith ${ }^{1}$, Antoine Berry ${ }^{2}$, Jean-François Allienne ${ }^{1}$, Judith Fillaux ${ }^{2}$, غ̇ve Toulza ${ }^{1}$
${ }^{1}$ Université Perpignan Via Domitia, IHPE UMR 5244, CNRS, IFREMER, université Montpellier, F-66860 Perpignan, France.

${ }^{2}$ Service de parasitologie-mycologie, CHU de Toulouse, Toulouse, France.

boissier@univ-perp.fr
> La schistosomiase, ou bilharziose, est une maladie parasitaire tropicale et subtropicale causée par des parasites qui habitent les vaisseaux sanguins humains et sont transmis par des mollusques d'eau douce spécifiques. L'infection humaine se produit par la pénétration transcutanée du stade larvaire infectieux du parasite émis par le mollusque vecteur, dans l'eau de baignade. En raison du nombre important de personnes infectées (plus de 200 millions dont $85 \%$ en Afrique) et du taux élevé de mortalité qu'elle induit (200000 décès par an), cette maladie est une priorité pour l'Organisation mondiale de la santé (OMS). Parmi les sept espèces de schistosome impliquées en santé humaine, Schistosoma haematobium, responsable de la bilharziose uro-génitale, est connu pour n'infecter que l'homme. Cette caractéristique d'être non zoonotique, ainsi que les températures élevées nécessaires au développement des stades larvaires du parasite dans le mollusque vecteur, peuvent expliquer l'absence de cette pathologie dans la liste des maladies parasitaires présentant un risque d'introduction en Europe.

Un foyer de bilharziose en France : une situation inédite

En janvier 2014, un jeune garçon de 12 ans se présentait à l'hôpital de Düsseldorf pour une hématurie ${ }^{l}$. Des œufs de Schistosoma haematobium furent alors retrouvés dans ses urines [1]. En

\footnotetext{
${ }^{1}$ Présence de sang dans l'urine.
}

mars 2014, 6 membres de 3 familles distinctes étaient diagnostiqués positifs à Schistosoma haematobium au centre hospitalier de Purpan, à Toulouse en France [2]. De manière surprenante, l'anamnèse ${ }^{2}$ des patients ne faisait pas état d'un voyage dans une zone endémique pour la bilharziose uro-génitale. Cependant, toutes les familles avaient séjourné, au cours de l'été 2013, dans le village de Sainte-Lucie de Porto-Vecchio (Corse du Sud, France). SainteLucie de Porto-Vecchio doit sont attractivité (3000 à 5000 personnes/jour y séjournent durant la haute saison) à la rivière du Cavu, succession de piscines naturelles dont l'eau atteint une température de $30^{\circ} \mathrm{C}$ en saison estivale (Figure IA). Une contamination par Schistosoma haematobium suppose la présence, dans l'eau, du mollusque vecteur. Notre laboratoire, centre collaborateur de I'OMS, a été mandaté par l'ANSES 3 pour mener les investigations afin d'identifier le vecteur dans la rivière. Comme d'autres cours d'eau de Corse qui avaient été prospectés au milieu du siècle dernier [3], la rivière du Cavu héberge de façon abondante Bulinus truncatus (Figure IB), le mollusque vecteur de Schistosoma haematobium. Le 16 mai 2014, une alerte sanitaire (rapid risk assessment) est donc publiée par le centre européen de prévention et de contrôle des maladies, l'ECDC (Euro-

\footnotetext{
${ }^{2}$ Interrogatoire réalisé par le médecin.

${ }^{3}$ Agence nationale de sécurité sanitaire de l'alimentation, de l'environnement et du travail.
}

pean centre for disease prevention and control), afin d'alerter les praticiens européens sur ce nouveau foyer de transmission. En France, environ 37000 dépistages sont réalisés entre mai 2014 et mai 2015 et un total de 125 cas sont aujourd'hui recensés.

Le parasite incriminé est une espèce hybride

L'examen microscopique des urines, à la recherche d'œufs de parasite, est la technique classique pour faire le diagnostic de la bilharziose uro-génitale. La plupart des œufs observés chez les patients du foyer corse ont une morphologie typique de Schistosoma haematobium (Figure 1C). Un seul patient présentait des œufs avec une morphologie atypique puisque caractéristique de Schistosoma bovis, l'agent de la bilharziose bovine. Afin de préciser ces observations, les œufs issus des patients ont été génotypés à l'aide de deux marqueurs moléculaires: un marqueur nucléaire (ITS2, ribosomal internal transcribed spacer 2) à héritabilité biparentale, et un marqueur mitochondrial (COI, cytochrome oxidase subunit I) uniquement transmis par la mère. Ces marqueurs sont classiquement utilisés pour l'identification des espèces de schistosome [4]. Outre la forme pure de $S$. haematobium, la moitié des œufs recueillis auprès des patients sont en fait des hybrides entre S. haematobium et $S$. bovis, l'espèce contaminant principalement le bétail [ 5 , 6]. De très rares œufs ont des génotypes purs similaires à $S$. bovis (révélés par les 


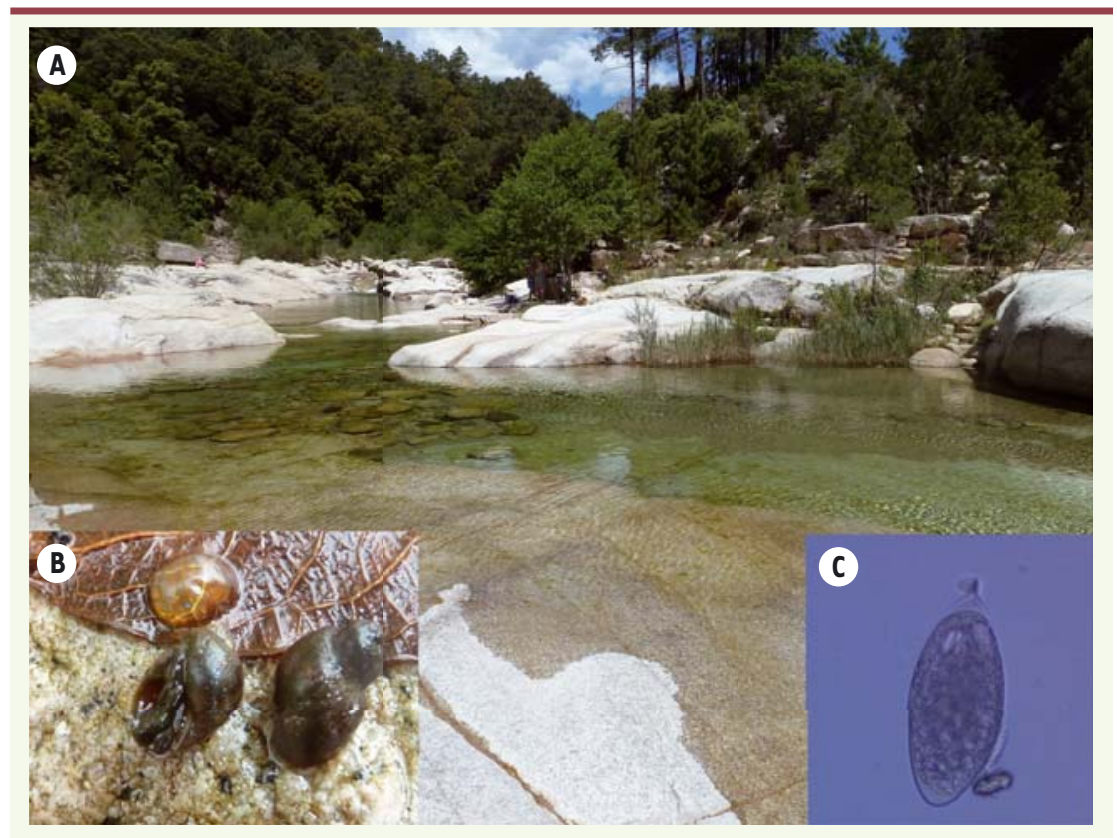

Figure 1. Un foyer de bilharziose et une espèce hybride. A. Piscine naturelle de la rivière du Cavu, en Corse. B. Bulinus truncatus, le mollusque vecteur de la bilharziose uro-génitale C. Fuf de Schistosoma haematobium isolé de l'urine des patients infectés en Corse.

marqueurs ITS2 et COI, correspondant à S. bovis). De tels parasites hybrides ont déjà été identifiés, dès 2009, au Sénégal [4], où ils sont largement répandus dans la population humaine $(88 \%$ des patients, localisés sur la quasi-totalité du territoire sénégalais, portent le parasite hybride) [7]. Des cas d'hybridations naturelles entre espèces de schistosome sont en fait de plus en plus souvent recensés chez l'homme: S. mansoni (agent de la bilharziose intestinale humaine) et $S$. rodhaini (agent de la bilharziose de rongeurs) au Kenya; S. mansoni et S. haematobium au Sénégal ; $S$. guineensis et $S$. haematobium au Bénin ou au Cameroun. Les hybridations entre espèces de Schistosoma rendent les situations épidémiologiques beaucoup plus complexes.

\section{Des hybrides aux capacités supérieures?}

L'hybridation permet au parasite d'élargir son spectre d'hôte intermédiaire et/ ou définitif. Par exemple, le croisement entre $S$. haematobium et $S$. intercalatum (agent de la bilharziose recto-sigmoï- dienne africaine) donne des résultats surprenants [8]. S. haematobium est capable d'infecter $32 \%$ des mollusques Bulinus truncatus mais il est incapable d'infecter Bulinus forskalii. À l'inverse, S. intercalatum peut infecter $40 \%$ des mollusques Bulinus forskalii mais il est incapable d'infecter Bulinus truncatus. L'hybride entre $S$. haematobium et $S$. intercalatum est capable, lui, d'infecter $B$. truncatus et $B$. forskalii dans respectivement $77 \%$ et $15 \%$ des cas. Le parasite hybride a donc à la fois accru ses capacités infectieuses et étendu son spectre d'hôtes possibles. Dans le cas de I'hybride formé entre S. haematobium et $S$. bovis, une situation similaire est à craindre. En effet, une augmentation des capacités infectieuses (appelée «hétérosis » ou «vigueur hybride») pourrait expliquer l'émergence de la bilharziose en Corse en conférant au parasite une capacité d'adaptation particulière lui permettant d'infecter une plus grande diversité de mollusques. B. truncatus est naturellement présent dans les îles méditerranéennes et dans tout le sud de l'Europe (France, Espagne, Portugal,
Italie, Grèce). Par ailleurs, le parasite S. bovis peut infecter des mollusques du genre Planorbarius. Or, Planorbarius metidjensis est largement répandu dans toute la péninsule ibérique. Toutefois, nous ne savons pas si ce mollusque peut abriter l'hybride formé entre $S$. haematobium et $S$. bovis qui infecte l'homme. Au-delà de l'hôte vecteur, c'est-à-dire du mollusque, on peut également se poser la question de la capacité de cet hybride à infecter les ruminants. Les études menées au Sénégal n'ont pas mis en évidence la présence de l'hybride dans les vaisseaux mésentériques de la vache (qui est le siège de S. bovis) [7]. Cependant, les auteurs n'ont pas analysé le système vasculaire de la vessie (siège de S. haematobium) ni les produits excrétés par les animaux (urine et/ ou fèces).

L'hybridation peut également augmenter la virulence du parasite vis-à-vis de son hôte vertébré. Expérimentalement, dans le modèle utilisant le hamster comme hôte définitif, $30 \%$ des cercaires ${ }^{4}$ de S. haematobium et $23 \%$ de S. intercalatum sont capables de se développer en parasite adulte. L'hybride entre $S$. haematobium et $S$. intercalatum voit sa capacité infectante augmenter à $66 \%$ [8]. La pathologie induite (qui se traduit par une hépatomégalie et une splénomégalie) est de ce fait accrue.

Enfin, l'hybridation entre les deux espèces de parasite peut compliquer le diagnostic. Les vers adultes de S. haematobium vivent dans les capillaires du plexus veineux péri-vésical, les œufs étant évacués par l'urine. $\varepsilon$ n revanche, on retrouve $S$. bovis dans les veines mésentériques et les œufs sont libérés par les fèces. Où sont donc évacués les œufs de la forme hybride formée entre espèces de parasite? Chez les schistosomes, la rencontre entre le mâle et la femelle se réalise au niveau de la veine porte. Les deux vers, accouplés, se dirigent ensuite

${ }^{4}$ Les cercaires sont la forme parasitaire qui infecte l'homme par voie transcutanée. 
vers le site de ponte, le mâle portant la femelle dans son canal gynécophore. On peut donc supposer que lors d'un croisement de première génération, le mâle d'une espèce conduit la femelle de l'autre espèce vers son propre site de reproduction. Pour les générations suivantes, le choix du site de ponte pourrait dépendre du niveau d'introgression génétique. Les études réalisées au Sénégal, portant sur des hybrides naturels de $n^{\text {ième }}$ génération, montrent que les œufs produits peuvent être évacués dans l'urine et/ ou dans les fèces [4]. En Corse, seules les urines ont été collectées et analysées pour le diagnostic parasitologique. Parmi 38 patients locaux (i.e. habitant la Corse) séropositifs pour le parasite, seuls 6 présentaient des œufs dans leurs urines. Pour les patients exempts d'œufs dans les urines, il est possible qu'ils n'aient été infectés que par des parasites du même sexe ou que la charge parasitaire était très faible. Nous ne pouvons cependant pas exclure la possibilité que les œufs de la forme hybride du parasite aient été évacués par les selles et donc non détectés.

L'émergence de la bilharziose en Corse est le premier exemple concret, en
Europe, de l'impact des changements globaux sur l'émergence de maladies infectieuses touchant d'autres continents. C'est la convergence entre les flux de populations humaines (tourisme, migrations) et l'augmentation de la température des eaux douces qui rend l'environnement propice à l'installation du cycle parasitaire (les températures des rivières corses ont augmenté de $1{ }^{\circ} \mathrm{C}$ au cours des 50 dernières années). De nombreuses questions se posent désormais: quelle est la capacité du schistosome hybride à s'adapter aux mollusques présents en Europe du Sud? Le parasite peut-il survivre tout au long de l'année sous nos latitudes? Quelle est sa capacité à se propager après qu'il se soit adapté à des hôtes réservoirs comme le bétail ? Quelle est la pathogénicité du schistosome hybride chez les humains ?... L'émergence de la bilharziose en Corse nous rappelle ainsi, qu'en plus des maladies à transmission vectorielle, transmises par les moustiques, comme la dengue ou le chikungunya, il faut également considérer les maladies transmises par les mollusques vecteurs. $\diamond$

Global changes and snail-borne

disease: an hybrid schistosome

reaches Europe

\section{LIENS D'INTÉREิT}

L'auteur déclare n'avoir aucun lien d'intérêt concernant les données publiées dans cet article.

\section{RÉFÉRENCES}

1. Holtfreter MC, Mone H, Muller-Stover I, et al. Schistosoma haematobium infections acquired in Corsica, France, August 2013. Euro Surveill $2014 ; 19$.

2. Berry $A$, Moné $H$, Iriart $X$, et al. Schistosomiasis haematobium, Corsica, France. Emerg Infect Dis $2014 ; 20: 1595-7$.

3. Doby JM, Rault B, Deblock S, Chabaud A. Snails and bilharziasis in Corsica. Distribution, frequency and biology of Bulinus truncatus. Ann Parasitol Hum Comp 1966 ; 41 : 337-49.

4. Huyse T, Webster BL, Geldof S, et al. Bidirectional introgressive hybridization between a cattle and human schistosome species. PLoS Pathog 2009 ; 5 : el000571.

5. Mone H, Holtfreter MC, Allienne JF, et al. Introgressive hybridizations of Schistosoma haematobium by Schistosoma bovis at the origin of the first case report of schistosomiasis in Corsica (France, Europe). Parasitol Res 2015 (sous presse).

6. Boissier J, Mone H, Mitta G, et al. Schistosomiasis reaches Europe. Lancet 2015 ; 15 : 757-8.

7. Webster BL, Diaw OT, Seye MM, et al. Introgressive hybridization of Schistosoma haematobium group species in Senegal: species barrier break down between ruminant and human schistosomes. PLOS Negl Trop Dis 2013; 7 : e2110.

8. Webster BL, Southgate VR. Compatibility of Schistosoma haematobium, S. intercalatum and their hybrids with Bulinus truncatus and $B$. forskalii. Parasitology 2003 ; 127 : 231-42.

\section{NOUVELLE}

\section{Sphingomab ${ }^{T M}$, un anticorps anti-sphingosine 1-phosphate, comme agent anti-hypoxique dans le cancer}

Olivier Cuvillier ${ }^{1-3}$
> La sphingosine 1-phosphate (SIP) est un lipide impliqué dans la régulation de la prolifération, la survie, la migration, et l'invasion cellulaires. II intervient également dans l'angiogenèse [1]. La SIP est le produit de la phosphorylation de la sphin- gosine par les sphingosine kinases. Bien que deux isoformes de sphingosine kinases ont été décrites, SphKl et SphK2, le rôle de la SphKl est beaucoup mieux caractérisé dans le cancer que celui de la SphK2 [2]. La SphKl, activée par de très nombreux
${ }^{1}$ Institut de pharmacologie et de biologie structurale, CNRS UMR 5089, BP 64182, 205 , route de Narbonne, 31077 Toulouse Cedex 4, France.

${ }^{2}$ Université de Toulouse, UPS, IPBS, Toulouse, France.

3 Équipe labellisée Ligue contre le Cancer. olivier.cuvillier@inserm.fr

agonistes (facteurs de croissance, hormones, cytokines, etc.), génère la SIP qui peut alors être sécrétée et agir de façon extracellulaire. Elle se lie à l'un de ses cinq récepteurs $\left(S_{1} P_{1-5}\right)$, qui sont des récepteurs couplés aux protéines $G$, induisant 